\section{§4. Open-Loop Excitation and Electrical Parameter Estimation of LHD Superconducting Coils}

Chikaraishi, H., Takami, S., Inoue, T., LHD Group, Ise, T. (Osaka Univ.)

In the LHD experiments regarding plasma current, the change of plasma current induced voltages to the coils and the coil current controller produced counter voltage to regulate the coil currents. These counter voltages induced a plasma one-turn voltage and affected the plasma current. This induced one-turn voltage depends on the current control scheme, and smaller one-turn voltage is expected in some plasma experiments. The use of an open loop-system, which involves no feedback of coil currents, is possible way to remove this effect. The design of such an open-loop system must take into account accurate electrical parameters of the inductance matrix and resistances of the coil system.

For this purpose, we estimated the cricuit resistance from the steady state controll error of the coil current. Next, we estimated inductance matrix of the LHD coil with following way. Figure 1 shows waveforms when the $\mathrm{HO}$ coil current reference was changed. In the figure, the current drift was rejected. The coil current change $\delta \mathbf{i}$ and its reference $\delta \mathbf{i}^{*}$ have a relation of $\mathbf{L} \delta \mathbf{i}=\mathbf{L}^{*} \delta \mathbf{i}^{*}$. When we use six different references, the inductance matrix can be estimated as $\mathbf{L}=\mathbf{L}^{*} \delta \mathbf{i}^{*} \delta \mathbf{i}^{-\mathbf{1}}$.

First, we measured the current drifts with open loop control to confirm the new estimated equivalent resistance. Figure 2 shows the current waveforms. For comparison, we set $\mathbf{v}^{*}$ to zero while $40<t<100$. All currents drifted in the same way when $\mathbf{v}^{*}$ was set to zero. In the open-loop controller with the new estimated resistances, the drift of the HM coil current was almost 0 $\mathrm{A}$, and the $\mathrm{HO}$ and $\mathrm{HI}$ currents drifted the opposite way. The drift of the average coil current was well compensated using the new estimated resistance. Small estimation errors remained in the resistances and lead the coil current unbalance, which are sensitive to voltage error. Even though small current drifts about 20 A during 60 s operation occur, they are sufficiently smaller than the operation current of $866 \mathrm{~A}$, and thus do not interfere with the power supplies.

Next, we tested the new inductance matrix. Figure 3 shows the test results using the previous inductance matrix and Figure 4 shows the results using the new matrix. With the new parameter, the turbulences of HM and HI coil currents were suppressed to $5 \mathrm{~A}$ as shown in Figure 4. With these results, the new estimated inductance matrix has a closer fit to the actual inductance matrix than the previous one. In both figures, the waveform of the $\mathrm{HO}$ coil current shows offset of $5 \mathrm{~A}$ and a small over-shoot when the current ramps down. These offset and over shoot are caused by the induced voltages due to the change of the eddy current flowing in the structure. When more accurate control is required for this control scheme, the effect of eddy current should be considered in the controller's design.

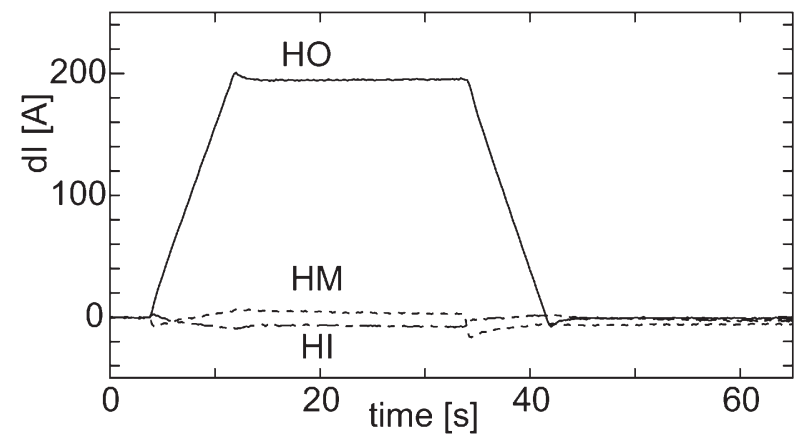

Figure 1. Current response using open loop controller

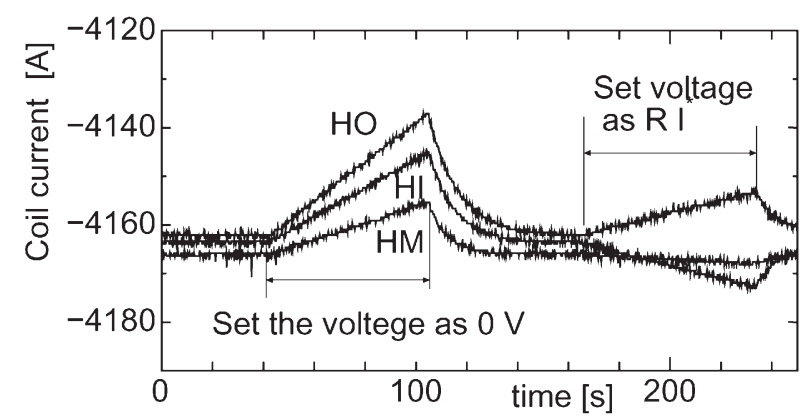

Figure 2. Current drift when open loop controller with new estimated parameters is used

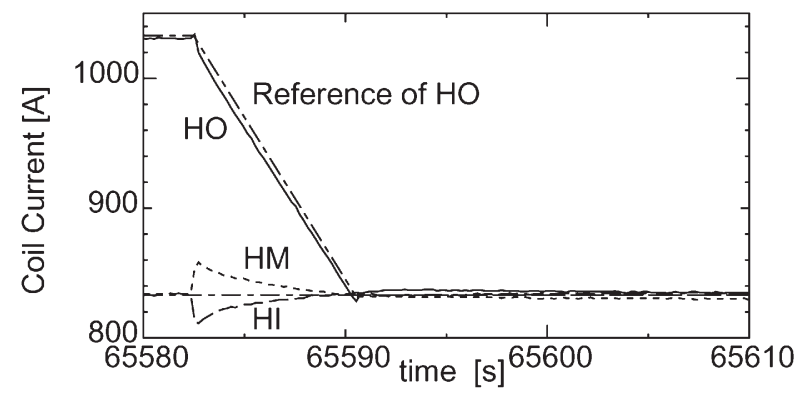

Figure 3. Current waveforms when the previous inductance matrix is used in the controller

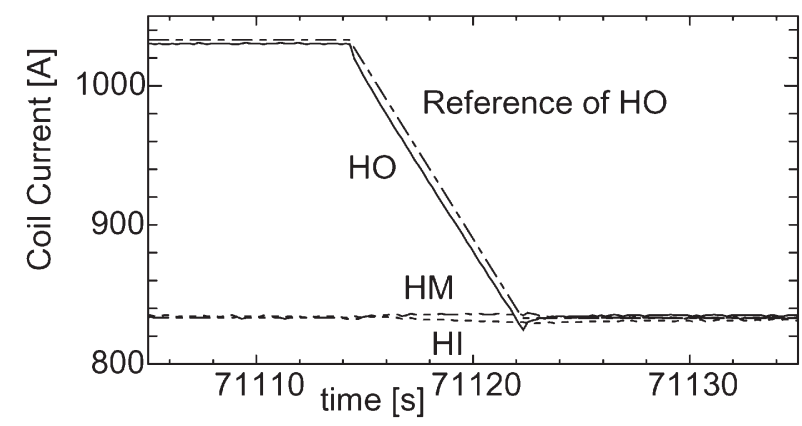

Figure 4. Current waveforms when the new estimated inductance matrix is used in the controller 